\title{
Clinical, laboratory and ultrasound characteristics of patients with primary hyperparathyroidism with renal involvement
}

\begin{abstract}
Milena Nikolova ${ }^{1}$, Georgi Ivanov $^{1}$, Tanya Tsocheva $^{1}$, Radina Nikolova ${ }^{1}$, Vladislava Milenova $^{1}$, Rumiana Krasteva ${ }^{1}$, Regina Djerassi ${ }^{1}$, Boris Bogov $^{1}$, Mihail Boyanov ${ }^{2}$, Yordan Vlahov ${ }^{3}$
\end{abstract}

1. Clinic of Nephrology, University Hospital Alexandrovska, Medical University, Sofia;

2. Clinic of Endocrinology, University Hospital Alexandrovska, Medical University, Sofia;

3. Clinic of Endocrinology, University Hospital Sofiamed, Medical Faculty, Sofia University;

\begin{abstract}
Primary hyperparathyroidism (PHPT) is unregulated hyperproduction of parathyroid hormone (PTH), inadequate to serum calcium levels. High PTH levels are associated with hypercalciemia, hypercalciuria and hyperphosphaturia, increased bone absorbtion with decreased bone mass and density, gastric hyperacidity with the development of peptic ulcers, and tissue deposition of calium phosphate with pancreatitis, nephrocalcinosis and vascular calcifications. The kidney involvement in PHPT is associated with: nephrolithiasis, nephrocalcinosis, chronic renal disease, and renal tubular dysfunction.
\end{abstract}


We present a series of 12 consecutive patients (4 male and 8 female, mean age 52.8 +/- 15.1 years, range 30-73) with kidney damage due to PHPT, admitted to the Clinic of Nephrology for the period May 2013 - September 2017. All patients had nephrolithiasis or history of nephrolithiasis, and nephrocalcinosis (cortical and/or medullary), with or without other organ involvement related to persistent hypercalciemia.

We discuss the current knowledge on the diagnosis and treatment of renal involvement in PHPT.

Keywords: primary hyperparathyroidism, renal involvement, hypercalciemia, nephrocalcinosis, nephrolithiasis, abdominal ultrasound, diagnosis, treatment.

\section{Introduction}

Primary hyperparathyroidism (PHPT) is unregulated hyperproduction of parathyroid hormone (PTH), inadequate to serum calcium levels $(1,2)$. In PHPT patients PTH is usually produced by parathyroid adenoma or parathyroid cancer cells, autonomous from the serum calcium and phosphate levels and from vitamin $\mathrm{D}$ serum levels. It usually presents between the age of 30 and 70 , with kidney stones, gastric hyperacidity, pancreatitis, or is detected due to abnormally high serum calcium and creatinine levels with or without renal stones. It is more frequent in women, female patients are usually older and symptomatic at presentation, whereas male are usually asymptomatic and slightly younger (3). In some cases MEN1 (multiple endocrine neoplasia type 1 , characterized by the development of tumors of $2 / 3$ principal endocrine organs - anterior pituitary, parathyroid glands and the entero-pancreatic endocrine tissue, and of non-endocrine tumors) syndrome is present (4).

High PTH levels are associated with:

- hypercalciemia - from mild to severe, even life-threatening,

- hypercalciuria (although normo-, even hypocalciuric patients have been described) and hyperphosphaturia with the formation of kidney stones,

- increased bone absorption with decreased bone mass and density (i.e., the classical "stone and bone" disease with the formation of kidney stones and/or calcifications plus bone lesions osteoporosis, bone fractures, osteitis fibrosa et cystic / osteoclastomas),

- increased synthesis of $1,25(\mathrm{OH}) 2 \mathrm{D}$, and increased intestinal absorption of calcium,

- gastric hyperacidity with the development of peptic ulcers, and

- tissue deposition of calcium phosphate with pancreatitis, nephrocalcinosis and vascular calcifications with or without end-organ ischemic damage. 
The kidney involvement in PHPT is associated with (Baradaran A): nephrolithiasis, nephrocalcinosis, chronic renal disease, and renal tubular dysfunction.

The prevalence of nephrolithiasis in PHPT patients has been shown to decrease - from $40-60 \%$ in the 1970 s-80s to $10-20 \%$ in the last two decades $(2,5)$. It should be noted that renal stones occur with the same frequency in normo- and hypercalciemic PHPT patients (15\% vs $19 \%)(6)$. In about $16 \%$ of the cases with PHPT kidney stones are bilateral (7). The surgical removal of the PTH-producing tumor has been shown to decrease the recurrence of nephrolithiasis in these patients $(2,5,8)$. Moreover, the presence of kidney stones is an indication for removal of the autonomous PTH-producing focus (2). Usually the kidney stones in PHPT consist of calcium oxalate and calcium phosphate. PHPT individuals with renal stones are usually hypercalciuric, but less than a third of the hypercalciuric PHPT patients develop kidney stones, and today it is impossible to predict which PHPT individuals will develop kidney stones based only on the amount on calciuria.

The exact mechanisms explaining the kidney stone formation in PHPT are not well understood. PTH is known to inhibit proximal tubular bicarbonate reabsorption and lead to mild metabolic acidosis (proximal tubular acidosis with high potassium levels). However, this is usually antagonized by the release of alkali due to fast bone resorption and the tubular reabsorption of bicarbonate due to hypercalcemia. Moreover, the calcium in the glomeruli in tubuli increases proportionately with hypercalcemia. Hypercalciuria and hyperphosphaturia plus the changes in bicarbonate urine levels are the major factors for kidney stone formation. Following risk factors are known to increase the risk of stone formation in PHPT: gender (male), age (more frequent in younger patients. High levels of 1,25(OH)2D (1).

Nephrocalcinosis is the deposition of calcium-containing salts in the renal parenchyma - renal tubular epithelial cells, tubular lumini, interstitial spaces, vascular walls. It is seen in patients with PHPT in the form of medullary, cortical and mixed medullary plus cortical nephrocalcinosis, with or without concomitant nephrolithiasis. The prevalence of nephrocalcinosis and nephrolithiasis in PHPT patients is unknown. In PHPT patients with parathyroid cancer the combined prevalence of both conditions is around $26 \%$ (8).

The development of renal failure in PHPT is usually related to the severity and duration of hypercalciuria, i.e. to the tubule-interstitial damage $(1,5)$. Chronic kidney disease with estimated glomerular filtration rate below $60 \mathrm{ml} / \mathrm{min}$ has been described in $13-16 \%$ of the PHPT patients in different series (5). Several risk factors have been shown to predict the development of renal failure in PHPT patients (5): age (50-70 years), gender (more frequently in females), dehydration, the presence of kidney stones and / or cysts, persistent and chronic elevation of PTH over time, insulin resistance (associated with PHPT and leading to hyperfiltration and endothelial dysfunction and faster nephrone loss), obesity (also leading to persistent hyperfiltration), poorly controlled arterial hypertension. Parathyroidectomy has been shown to ameliorate kidney function, yet the major determinants for the persistence of chronic kidney disease after the operation are the blood pressure and the pre-operative renal function (5).

Renal tubular dysfunction in PHPT is associated with: bicarbonate loss and the development of proximal tubular acidosis, calcium-induced tubular toxicity (including nephrocalcinosis), osmotic diuresis in hypercalciuria and dehydration, urinary tract infections in patients with kidney stones. 


\section{Materials and methods}

For the period May 2013 - September 2017 we observed 12 consecutive patients (4 male and 8 female, mean age 52.8 +/- 15.1 years, range 30-73) with kidney damage due to PHPT. All patients had nephrolithiasis or history of nephrolithiasis, and nephrocalcinosis (cortical and/or medullary), with or without other organ involvement related to persistent hypercalciemia, including peptic ulcers, pancreatitis and vascular disease.

All patients were taken medical and surgical history and underwent thorough physical examination. All patients were performed standard blood (including, blood count, biochemical, PTH levels and alkalinebase balance) and urine investigations and underwent abdominal ultrasound scan (standard investigation with ESAOTE My Lab 60, ultrasound probes 2.5-7 MHz), and (if needed) - X-ray imaging studies (including plain abdominal X-ray, intravenous pyelography and CT of the abdomen. In all patients the PHPT was proven with blood investigations (PTH levels) and biopsy-proven presence of parathyrioid adenoma (ultrasound-guided), and if needed - scintigraphic investigation and/or SPECT scan. Two patients had been diagnosed with MEN1 syndrome in adolescence (patients NN 1 and 5). None of the 12 patients had parathyroid cancer or ectopic PTH secretion.

Table 1. Demographic and clinical characteristics of 12 patients with PHPT with renal involvement.

\begin{tabular}{|c|c|c|c|c|c|c|c|c|c|c|c|c|}
\hline $\mathrm{N}$ & Gender & $\begin{array}{l}\text { Age } \\
\text { (y.) }\end{array}$ & $\begin{array}{l}\text { Renal } \\
\text { stones }\end{array}$ & $\begin{array}{l}\text { Renal } \\
\text { calcifications }\end{array}$ & $\begin{array}{l}\text { Peptic } \\
\text { ulcer }\end{array}$ & Pancreatitis & $\begin{array}{l}\text { Vascular } \\
\text { disease }\end{array}$ & $\begin{array}{l}\text { Calcium } \\
(\mathrm{mmol} / \mathrm{l})\end{array}$ & $\begin{array}{l}\text { Phosphate } \\
(\mathrm{mmol} / \mathrm{l})\end{array}$ & $\begin{array}{l}\mathrm{PTH} \\
(\mathrm{pmol} / \mathrm{l})\end{array}$ & $\begin{array}{l}\text { ALP } \\
\text { (U/l) }\end{array}$ & $\begin{array}{l}\text { Creatinine } \\
(\mathrm{mcmol} / \mathrm{l})\end{array}$ \\
\hline $1^{*}$ & $\mathrm{M}$ & 35 & + & + & - & - & - & 2,6 & 0,95 & 25.50 & 238 & 99 \\
\hline 2 & $\mathrm{M}$ & 65 & + & + & + & + & + & 2,98 & 0,68 & 122.50 & 466 & 150 \\
\hline 3 & $\mathrm{M}$ & 36 & + & + & - & - & - & 2,59 & 0,89 & 130.00 & 255 & 90 \\
\hline 4 & M & 30 & + & + & + & - & - & 2,77 & 0,77 & 99.80 & 208 & 95 \\
\hline $5^{*}$ & $\mathrm{~F}$ & 35 & + & + & - & - & - & 2,66 & 0,84 & 88.90 & 225 & 87 \\
\hline 6 & $\mathrm{~F}$ & 60 & + & + & + & + & - & 3,34 & 0,75 & 130.20 & 556 & 145 \\
\hline 7 & $\mathrm{~F}$ & 58 & + & + & - & - & - & 2,64 & 0,90 & 124.00 & 199 & 133 \\
\hline 8 & $F$ & 73 & + & + & - & - & + & 3,55 & 0,69 & 99.70 & 183 & 142 \\
\hline 9 & F & 56 & + & + & - & - & - & 2,80 & 0,81 & 115.00 & 334 & 67 \\
\hline 10 & $\mathrm{~F}$ & 67 & + & + & + & + & + & 3,57 & 0,83 & 180.00 & 223 & 166 \\
\hline 11 & $\mathrm{~F}$ & 52 & + & + & - & - & - & 2,51 & 0,97 & 72.00 & 200 & 102 \\
\hline 12 & $\mathrm{~F}$ & 57 & + & - & - & - & + & 2.7 & 1.01 & 56.50 & 223 & 355 \\
\hline & & & & & & & & & & & & \\
\hline
\end{tabular}


The statistical analysis (descriptive and non-parametric comparative analysis) was performed for standard SPSS 20.0 for Windows.

The demographic and clinical characteristics of the presented patients are shown in table 1.

\section{Results}

We observed 12 patients with PHPT with renal involvement. The female : male ratio was 2:1, and in female patients the mean age was slightly higher, as were serum creatinine and calcium levels. None of the patients had parathyroid cancer or ectopic PTH secretion. All patients had nephrolithiasis and renal calcifications (medullary and/or cortical nephrocalcinosis). In 4 patients the renal involvement was the first manifestation that lead to the diagnosis PHPT.

Overall 7 patients had undergone ESWL (extracorporal sound wave lithotripsy) for renal stones; all 12 patients had history of renal colics and urinary tract infections.

Peptic ulcers had 4 patients, pancreatitis -3 , vascular disease had 4 (including 2 patients with angina pectoris and coronary stent implantation, 1 patient with history of stroke an 1 with history of myocardial infarction). One patient had diabetes (patient N12). Seven patients had arterial hypertension (patients N 2 , $6,7,8,10,11,12)$. At presentation, 7 patients had renal failure, one of them subsequently went on hemodialysis (patient N12). At the time of the first admission to the clinic 7 patients had hypercalciemia, 2 were normocaciemic and 3 had borderline serum calcium. Four patients had hypophosphatemia. PTH and ALP levels were increased in all patients. Four patients had alkaline-base disturbances - mild compensated metabolic acidosis (patients 6, 7, 8 and 12). The mean levels of some laboratory parameters are presented in table 2 .

Table 2. Mean values of some clinical-laboratory parameters of the investigated patients.

\begin{tabular}{|l|c|c|c|c|}
\hline & Mean & SD & Minimum & Maximum \\
\hline Creatinine (mcmol/l) & 134.25 & 70.24 & 67.0 & 335.0 \\
\hline Calcium (mmol/l) & 2.89 & 0.38 & 2.51 & 3.57 \\
\hline Phosphate (mmol/l) & 0.84 & 0,11 & 0.68 & 1.01 \\
\hline ALP (U/l) & 275.83 & 117.92 & 183.0 & 556.0 \\
\hline PTH (pmol/l) & 103.68 & 40.12 & 25.50 & 180.0 \\
\hline
\end{tabular}

The abdominal ultrasound of the investigated patients revealed nephrolithiasis and nephrocalcinosis medullary, cortical or combined medullary + cortical (figures 1, 2 and 3). In all patients renal stones were 
X-ray positive (i.e., calcium containing). All patients had history of renal colics and urinary tract infections. Figure 1 shows ultrasound examination of the neck in a patient with adenoma of the left inferior parathyroid gland.

Figure 1. Medullary nephrocalcinosis and nephrolithiasis (patient N6). Note the classical "garlandlike" hyperechogenic zone around the pyramids (arrow) and the contrasting hypoechogenic pyramids.

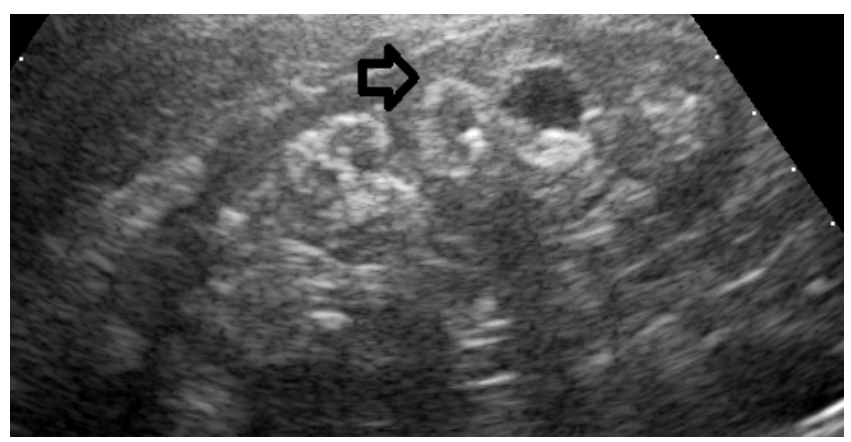

Figure 2. Medullary and cortical nephrocalcinosis and nephrolithiasis, (patient N2).

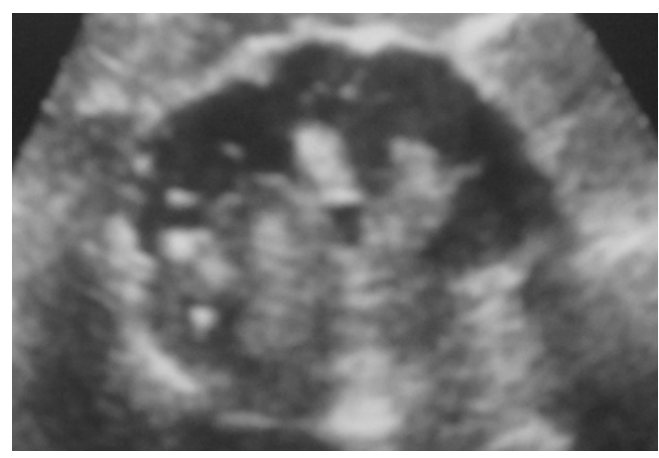

Figure 3. Medullary calcifications (arrow) (patient N11).

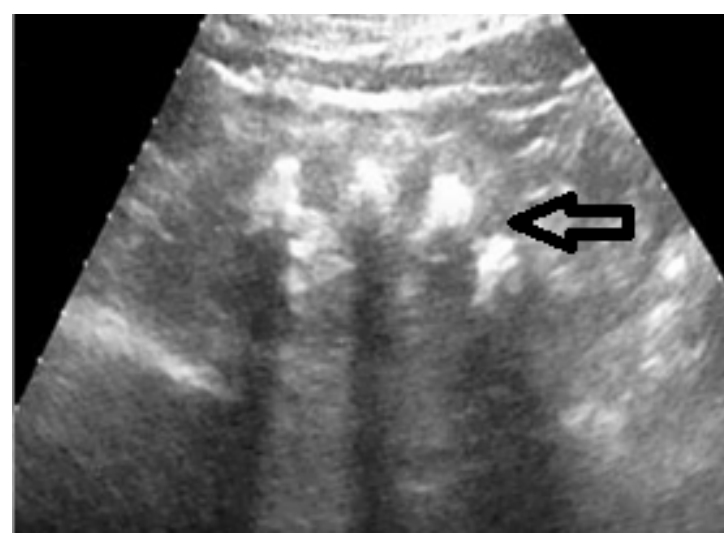


Figure 4. Ultrasound examination of the neck in a patient with parathyroid adenoma and PHPT left inferior parathyropid gland (patient N6).

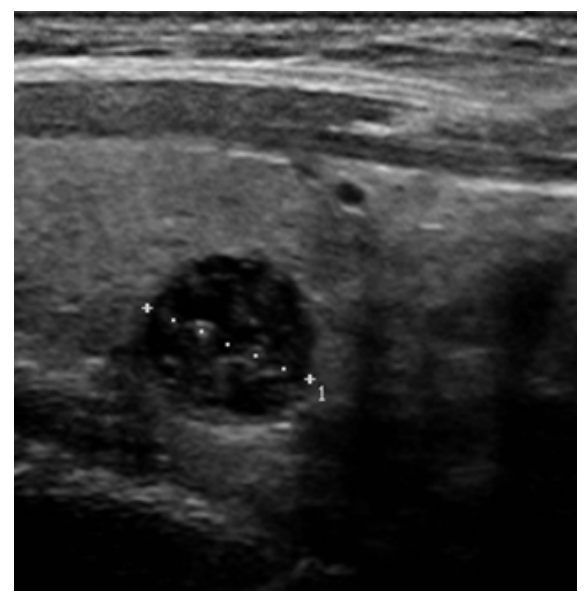

All investigated patients underwent parathyroidectomy and in all calcium, phosphate, PTH and ALP levels returned back to the normal limits. One patient went on hemodialysis treatment (patient N12) and one patient died of sepsis (patient N8).

The comparative analysis (table 3) showed that in our series men are slightly younger than women with PHPT, with slightly lower creatinine, calcium, phosphate and PTH levels and slightly higher ALP ( $p=n . s$. for all differences).

Table 3. Comparison between men and women with PHPT with renal involvement.

\begin{tabular}{|l|l|c|c|c|c|}
\hline \multirow{2}{*}{ Parameter } & Gender & N & Mean / frequency & SD & p \\
\hline \multirow{2}{*}{ Age } & male & 4 & 41.5 & 15.9 & n.s. \\
\cline { 2 - 6 } & female & 8 & 58.5 & 11.8 & \\
\hline \multirow{2}{*}{ Peptic ulcer } & male & 4 & $2 / 4$ & & n.s. \\
\cline { 2 - 7 } & female & 8 & $2 / 8$ & & \\
\hline \multirow{2}{*}{ Vascular disease } & male & 4 & $1 / 4$ & & n.s. \\
\cline { 2 - 7 } & female & 8 & $2 / 8$ & & n.s. \\
\cline { 2 - 7 } & female & 8 & $1 / 4$ & & \\
\hline \multirow{2}{*}{ Creatinine } & male & 4 & 108.5 & 27.9 & n.s. \\
\cline { 2 - 6 } & female & 8 & 147.1 & 82.8 & \\
\hline
\end{tabular}




\begin{tabular}{|l|l|c|c|c|c|}
\hline Calcium & male & 4 & 2.7 & 0.2 & n.s. \\
\cline { 2 - 6 } & female & 8 & 2.9 & 0.4 & \\
\hline \multirow{2}{*}{ Phosphate } & male & 4 & $0 ., 82$ & 0.12 & n.s. \\
\cline { 2 - 6 } & female & 8 & 0.85 & 0.10 & \\
\hline \multirow{2}{*}{ ALP } & male & 4 & 291.8 & 117.8 & n.s. \\
\cline { 2 - 6 } & female & 8 & 267.9 & 125.2 & \\
\hline \multirow{2}{*}{ PTH } & male & 4 & 94.5 & 47.7 & n.s. \\
\cline { 2 - 7 } & female & 8 & 108.3 & 38.5 & \\
\hline \multirow{2}{*}{ MEN } & male & 4 & $1 / 4$ & & n.s. \\
\cline { 2 - 6 } & female & 8 & $1 / 8$ & & \\
\hline
\end{tabular}

\section{Discussion}

PHPT is the third most common endocrine disorder (5). It is observed more frequently in women (3), between the age of 30 and 70 , usually asymptomatic for a long time, discovered mainly in association with renal involvement, peptic ulcer or pancreatitis or detected accidentally due to high serum calcium. For the period between May 2013 and September 2017 in our Clinic we observed a total of 12 patients with PHPT, in 4 of whom kidney involvement was the first manifestation of the disease. The majority of patients were women (8/12), aged $58.5+/-11.8$ years (i.e., in the fifth - seventh decade). The female : male ratio was 2:1 which is in accordance with the literature data for the higher prevalence of PHPT in women (3). All 12 patients were admitted to the Clinic for diagnostic evaluation of renal stones, renal parenchymal calcifications and/or increased serum creatinine. Moreover, women were slightly older than men, with slightly higher serum calcium and creatinine levels and PTH, with the differences not reaching statistical significance, probably due to the small number of patients. None of the patients had parathyroid cancer or ectopic PTH secretion.

In all patients we detected kidney stones (X-ray positive) and all had ultrasound data for nephrocalcinosis - calcifications within the medulla and/or the renal cortex - the typical ultrasound image of PHPT (figures 1, 2 and 3). Yet, the number of stones and the site and amount of calcifications in the renal tissue did not correlate with the levels of PHT or serum creatinine. Signs of tubular dysfunction had 4 patients and 7 patients had renal failure.

All patients had past history of renal colics and urinary tract infections.

Only one patient (with concomitant type 2 diabetes and arterial hypertension) went on dialysis during the follow-up and one patient died of sepsis.

The results of our study show that nephrolithiasis and nephrocalcinosis can be the first manifestation of PHPT. Moreover, PHPT with renal involvement is more frequent in females around their sixth decade, with 
slightly impaired renal function and high serum calcium levels. Therefore, all patients with recurrent calcium-containing renal stones, especially with nephrocalcinosis and increased serum calcium and/or low phosphate levels, should be investigated for PHPT (serum PTH levels, ultrasound examination of the neck and, if needed, further imaging studies, including scintigraphy and/or SPECT scan).

\section{References}

1. Baradaran A. Primary hyperparathyroidism and kidney: recent findings. J Parathyroid Dis 2014;2(1):7-8.

2. Lila AR, Sarathi $V$, Jagtap $V$, et al. Renal manifestations of primary hyperparathyroidism. Indian J Endocrinol Metab. 2012 Mar-Apr; 16(2): 258-262.

3. Mazeh H, Sippel RS, Chen $\mathrm{H}$. The role of gender in primary hyperparathyroidism: same disease, different presentation. Ann Surg Oncol. 2012 Sep;19(9):2958-62.

4. Giusti F, Tonelli F, Brandi ML. Primary hyperparathyroidism in multiple endocrine neoplasia type 1: when to perform surgery? Clinics 2012;67(S1):141-144.

5. Verdelli C, Corbetta S. Mechanisms in Endocrinology. Kidney involvement in patients with primary hyperparathyroidism: an update on clinical and molecular aspects. Eur J Endocrinol 2017;176 (1):R39-R52.

6. Tuna MM, Caliskan M, Unal M, et al. Normocalcemic hyperparathyroidism is associated with complications similar to those of hypercalcemic hyperparathyroidism. Journal of Bone and Mineral Metabolism 201634 331-335.

7. Cipriani C, Biamonte F, Costa AG, Zhang et al. Prevalence of kidney stones and vertebral fractures in primary hyperparathyroidism using imaging technology. Journal of Clinical Endocrinology and Metabolism 2015100 1309-1315.

8. Rejnmark L, Vestergaard P, Mosekilde L. Nephrolithiasis and renal calcifications in primary hyperparathyroidism. J Clin Endocrinol Metab 2011;96:2377-2385.

\section{Corresponding author:}

Dr. Milena Nikolova, MD, PhD

Clinic of Nephrology,

University Hospital Alexandrovska,

Medical University

1 G. Sofiiski Str., 1431

Sofia, Bulgaria

email: milena_i_dani@abv.bg 